\title{
Structural Rearrangements in R432A Variant of AAV2 Affect Genome Packaging
}

Lauren M. Drouin ${ }^{1}$, Bridget Lins ${ }^{1}$, Mandy Janssen ${ }^{3}$, Antonette Bennett ${ }^{1}$, Paul R. Chipman ${ }^{1}$, Robert McKenna ${ }^{1}$, Weijun Chen ${ }^{2}$, Nicholas Muzyczka ${ }^{2}$, Giovanni Cardone ${ }^{3}$, Timothy S. Baker ${ }^{3}$, and Mavis Agbandje-McKenna ${ }^{1}$

${ }^{1 .}$ Department of Biochemistry \& Molecular Biology, Center for Structural Biology, The McKnight Brain Institute, College of Medicine, University of Florida, Gainesville, FL, USA

2. Department of Molecular Genetics and Microbiology, University of Florida Genetics Institute, College of Medicine, University of Florida, Gainesville, FL, USA

3. Department of Chemistry and Biochemistry, and Division of Biological Sciences, University of California—San Diego, San Diego, California, USA

Adeno-associated viruses (AAVs) are promising therapeutic gene delivery vectors because they can package foreign genes and achieve stable, long-term gene expression in a broad range of tissues, with no known pathogenicity [1]. AAVs are small, non-enveloped, $\mathrm{T}=1$ icosahedral viruses that package singlestranded DNA [2]. Three structural proteins VP1, VP2, and VP3 share a common VP3 region. The unique VP1 and VP1/2 common regions contain signals required for viral infection. AAVs package their genomes into preformed capsids in the nucleus through the use of Rep proteins. However, this mechanism is poorly understood and the capsid determinants of successful genome packaging are not well characterized. A better understanding of this mechanism may aid in the improvement of AAV packaging efficiency, which currently only yields about one genome-packaged particle for every ten empty capsids in vector preparations [3]. To elucidate the capsid determinants of genome packaging, we used electron cryo-microscopy and three-dimensional image reconstruction methods to examine structural differences between wild-type (wt) AAV2 and a packaging deficient variant, AAV2-R432A. The single residue change, R432A, was identified in a charge-to-alanine mutagenesis screen as having a severe genome-packaging defect compared to wt-AAV2 [4]. This site is not accessible from the exterior surface of the capsid, where it could potentially interact with Rep, nor is it accessible from the interior, where it could interact with the packaged DNA. Thus, the role of residue 432 in genome packaging is difficult to reconcile. AAV2-R432A was also reported to have its VP1 and VP2 N-termini exposed abnormally on the capsid surface at room temperature, in contrast to wt-AAV2 [5]. It was postulated that the VP N-termini of AAV2-R432A are extruded through the 5-fold pores, one of which is proposed to be the portal for genome packaging [5], thus blocking the route for DNA encapsulation. However, chimeric AAV2 variants with externalized VP1 and VP2 still maintain wt packaging efficiencies [6]. Therefore, if the 5-fold channel functions as the packaging portal, perhaps not all twelve channels are blocked by extruded N-termini, suggesting a different mechanism of action for AAV2-R432A.

We used COOT [7] and the known AAV2 crystal structure coordinates (PDBID: 1LP3) as a template, to build models into cryo-EM density maps of wt-AAV2 and AAV2-R432A at resolutions of 3.8 and 3.7A, respectively. The models demonstrate that removal of the arginine side-chain in AAV2-R432A eliminates hydrogen-bonding interactions resulting in altered intra- and intermolecular interactions propagating from under the 3-fold axis towards the 5-fold channel. Also, structural rearrangements in AAV2-R432A lead to repositioning of the $\beta$ A strand away from its anti-parallel interactions with the $\beta B$ strand in wt to a new location with many fewer intramolecular interactions under the icosahedral 2-fold axis. Consistent with these observations, differential scanning calorimetry experiments reveal an $\sim 10^{\circ} \mathrm{C}$ decrease in thermal stability for AAV2-R432A compared to wt-AAV2. The AAV2-R432A structure 
reveals a buildup of density underneath the 2-fold axis, suggested to occur with the VP1/2 common region and VP1u N-terminus prior to extrusion through the 5-fold pore [8]. Density proximal to a region reported to be a nucleotide binding site [9] was observed in wt-AAV2 but not AAV2-R432A, consistent with the inability of the variant to package DNA. These data show that the 2-, 3-, and 5-fold regions of the capsid contribute to producing the packaging defect and highlight a tight connection between the entire capsid in maintaining packaging efficiency. Finally, the global effect of the single ARG to ALA residue change at buried position 432 , highlights the plasticity of the assembled capsid and the need to conserve structural fidelity in all functions, including capsid stability and genome packaging [10].

References:

[1] Asokan A, Scaffer DV, Samulski RJ, Mol Ther. 20 (2012): 699-708

[2] Chapman M, Agbandje-McKenna M, in "Parvoviruses", ed. Ker JR et al, Edward Arnold Ltd, NY

[3] Grimm D., et al., Gene Ther. 6 (1999):1322-1330.

[4] Wu P. et al., J Virol. 74 (2000): 8634-8647

[5] Bleker S, Pawlitta M, Kleinschmidt JA, J Virol. 80 (2006): 810-820

[6] Warrington et al., J Virol. 78 (2004): 6595-6609

[7] Emsley P. et al., Acta Cryst. D., 66 (2010): 486-501

[8] Kronenberg S. et al., J. Virol. 79 (2005): 5296-5303

[9] Nam H.J. et al., J. Virol. 81 (2007): 12260-12271

[10] Funding provided by NIH P01 HL51811, R01 AI1081961, R-37 GM033050, Training Grant T32GM008799, support from the University of California San Diego, and the Agouron Foundation to establish the cryoTEM facilities used in this study.
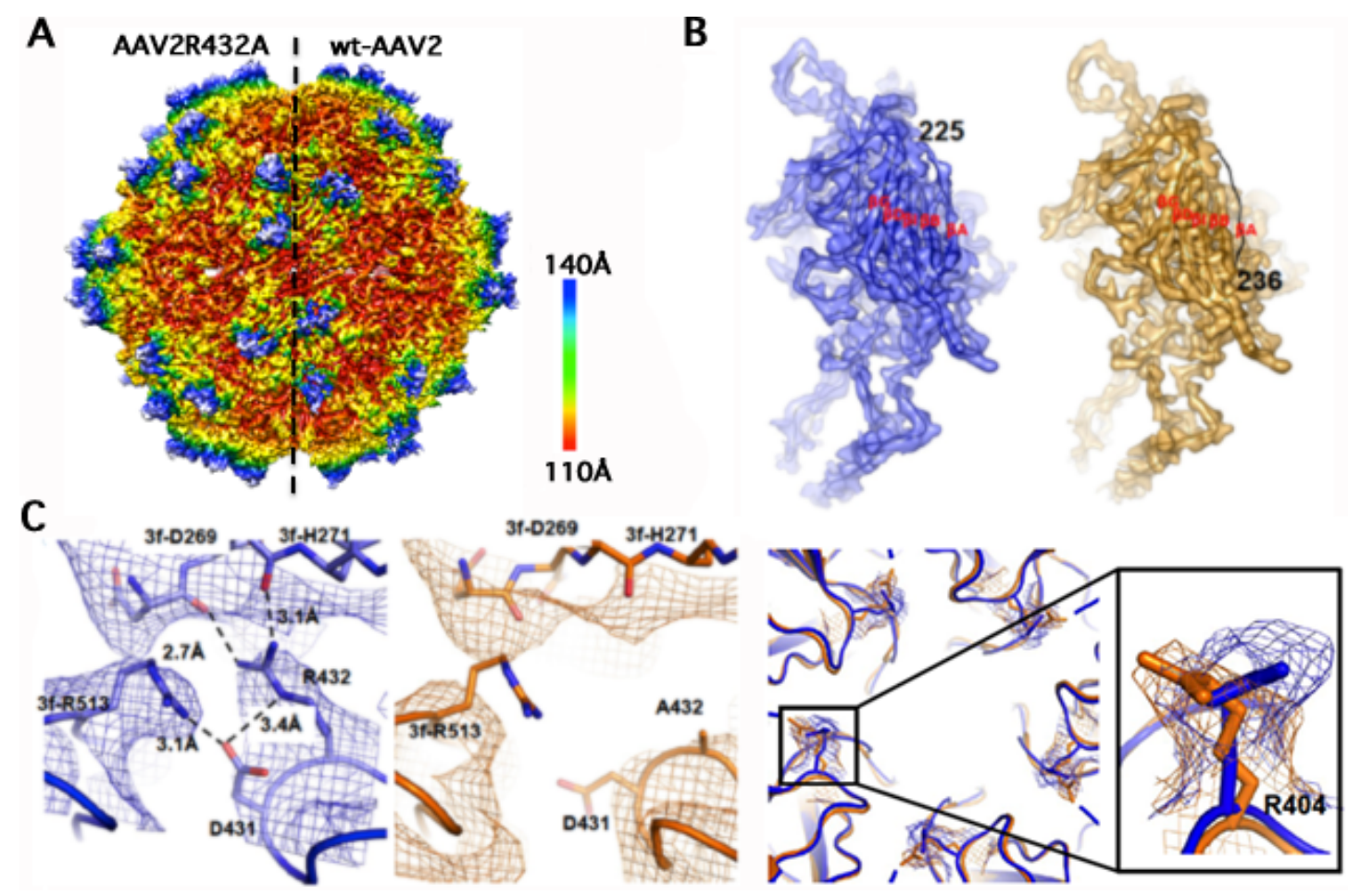

Figure 1. A) Radially color-cued cryoEM maps of AAV2-R432A (left) and wt-AAV2 (right). B) Density maps of wt-AAV2 (blue) and AAV2-R432A (gold) monomers viewed from the capsid interior. The AAV2 crystal structure (PDBID: 1LP3) is docked into each map and reveals the absence of the $\beta A$ strand in AAV2-R432A. C) Close-up views of the structural rearrangements in the vicinity of residue 432 in wt-AAV2 (left) and AAV2R432A (middle) and around residue 404 near the 5-fold axis (right and inset). 